\title{
GPDs at HERA and perspectives at COMPASS
}

\author{
Laurent SCHOEFFEL ${ }^{* \dagger}$ \\ CEA Saclay, Irfu/SPP, France \\ E-mail: laurent.schoeffelecea.fr
}

\begin{abstract}
Measurements of the deep-inelastic scattering (DIS) of leptons and nucleons, $e+p \rightarrow e+X$, allow the extraction of Parton Distribution Functions (PDFs) which describe the longitudinal momentum carried by the quarks, anti-quarks and gluons that make up the fast-moving nucleons. While PDFs provide crucial input to perturbative Quantum Chromodynamic (QCD) calculations of processes involving hadrons, they do not provide a complete picture of the partonic structure of nucleons. In particular, PDFs contain neither information on the correlations between partons nor on their transverse motion. Hard exclusive processes, in which the nucleon remains intact, have emerged in recent years as prime candidates to complement this essentially one dimentional picture. The simplest exclusive process is the deeply virtual Compton scattering (DVCS) or exclusive production of real photon, $e+p \rightarrow e+\gamma+p$. This process is of particular interest as it has both a clear experimental signature and is calculable in perturbative QCD. The DVCS reaction can be regarded as the elastic scattering of the virtual photon off the proton via a colourless exchange, producing a real photon in the final state $[1,2]$. In the Bjorken scaling regime, QCD calculations assume that the exchange involves two partons, having different longitudinal and transverse momenta, in a colourless configuration. These unequal momenta or skewing are a consequence of the mass difference between the incoming virtual photon and the outgoing real photon. This skewedness effect can be interpreted in the context of generalised parton distributions (GPDs) [3]. In this proceeding, we examine typical measurements from HERA and prospects for COMPASS at CERN [4], that can bring new insights on the quarks/gluons imaging of the nucleon.
\end{abstract}

European Physical Society Europhysics Conference on High Energy Physics

July 16-22, 2009

Krakow, Poland

* Speaker.

${ }^{\dagger}$ A footnote may follow. 


\section{Introduction}

A major experimental achievement of H1 and ZEUS [1,2] has been the measurement of DVCS cross sections, differential in $t=\left(p^{\prime}-p\right)^{2}$, the momentum transfer (squared) at the proton vertex. A good description of $d \sigma_{D V C S} / d t$ by a fit of the form $e^{-b|t|}$ is obtained [1,2]. Hence, an extraction of the $t$-slope parameter $b$ is accessible and it can be achieved experimentally. Measurements at HERA and simulations for the COMPASS kinematics are displayed in Fig. 1 [4]. We observe the good complementarity of both kinematical coverage in $x_{B j}$.

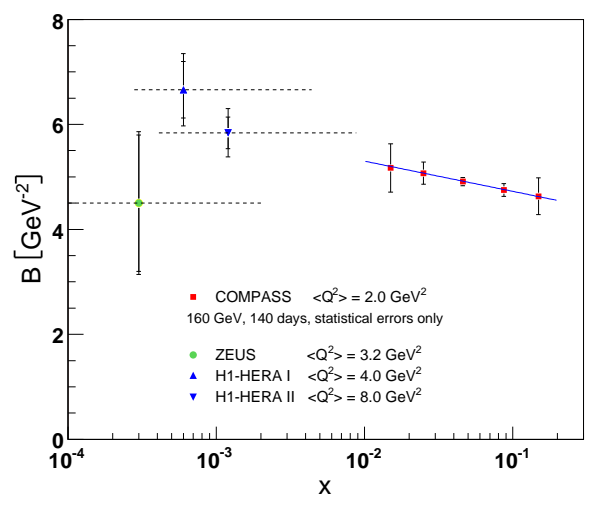

Figure 1: The logarithmic slope of the $t$ dependence for DVCS exclusive production, $b$ as a function of $x_{B j}$, extracted from a fit $d \sigma / d t \propto \exp (-b|t|)$ where $t=\left(p-p^{\prime}\right)^{2}$. H1 and ZEUS points are measurements and simulations are displayed for COMPASS (CERN).

\section{On the interest of $t$ slope measurement}

Measurements of the $t$-slope parameters $b$ are key measurements for almost all exclusive processes, in particular DVCS. Indeed, a Fourier transform from momentum to impact parameter space readily shows that the $t$-slope $b$ is related to the typical transverse distance between the colliding objects [3]. At high scale, the $q \bar{q}$ dipole is almost point-like, and the $t$ dependence of the cross section is given by the transverse extension of the gluons (or sea quarks) in the proton for a given $x_{B j}$ range. More precisely, from GPDs, we can compute a parton density which also depends on a spatial degree of freedom, the transverse size (or impact parameter), labeled $R_{\perp}$, in the proton. Both functions are related by a Fourier transform

$$
P D F\left(x, R_{\perp} ; Q^{2}\right) \equiv \int \frac{d^{2} \Delta_{\perp}}{(2 \pi)^{2}} e^{i\left(\Delta_{\perp} R_{\perp}\right)} G P D\left(x, t=-\Delta_{\perp}^{2} ; Q^{2}\right) .
$$

Thus, the transverse extension $\left\langle r_{T}^{2}\right\rangle$ of gluons (or sea quarks) in the proton can be written as

$$
\left\langle r_{T}^{2}\right\rangle \equiv \frac{\int d^{2} R_{\perp} P D F\left(x, R_{\perp}\right) R_{\perp}^{2}}{\int d^{2} R_{\perp} P D F\left(x, R_{\perp}\right)}=4 \frac{\partial}{\partial t}\left[\frac{G P D(x, t)}{G P D(x, 0)}\right]_{t=0}=2 b
$$


where $b$ is the exponential $t$-slope. Measurements of $b$ presented in Fig. 1 for HERA corresponds to $\sqrt{r_{T}^{2}}=0.65 \pm 0.02 \mathrm{fm}$ at large scale $Q^{2}$ for $x_{B j}<10^{-2}$. This value is smaller that the size of a single proton, and, in contrast to hadron-hadron scattering, it does not expand as energy $W$ increases. Obviously, it would be interesting to perform similar measurements in the COMPASS kinematic domain to obtain the information at intermediate $x_{B j}$ [4].

Let us note that HERA results are consistent with perturbative QCD calculations in terms of a radiation cloud of gluons and quarks emitted around the incoming virtual photon. The fact the perturbative QCD calculations provide correct descriptions of $b$ measurements is a proof that they deal correctly this non-trivial aspect of the proton (spatial) structure. The modeling of the correlation between the spatial transverse structure and the longitudinal momenta distributions of partons in the proton is one of major challenges for the GPDs approach.

Another natural way to address the problem of the correlation between $x$ and $t$ kinematical variables proceeds from a determination of a cross section asymmetry with respect to the beam charge. It has been performed recently by the $\mathrm{H} 1$ experiment by measuring the ratio $A_{C}=\left(d \sigma^{+}-\right.$ $\left.d \sigma^{-}\right) /\left(d \sigma^{+}+d \sigma^{-}\right)$as a function of $\phi$, where $\phi$ is the azimuthal angle between leptons and proton plane. The result is presented in Ref. [1]. $A_{C}$ is found to be

$$
A_{C} \simeq 0.16 \cos \phi
$$

at low $x_{B j}<0.01$. This result represents a major experimental progress and is challenging for models. Let us note that models of GPDs can use present HERA data at low $x_{B j}$, as well as JLab and HERMES data at larger $x_{B j}\left(x_{B j}>0.1\right)$, in order to provide a first global understanding of exclusive real photon production [3]. However, as already mentioned above, some efforts have still to be made in the intermediate $x_{B j}$ domain [4].

\section{Summary and outlook}

DVCS measurements in the HERA kinematics at low $x_{B j}\left(x_{B j}<0.01\right)$ are well described by recent GPDs models, which also describe correctly measurements at larger values of $x_{B j}$ in the JLab kinematics. Recently, H1 and ZEUS experiments have also shown that proton tomography at low $x_{B j}$ enters into the experimental domain of high energy physics, with a first experimental evidence that gluons are located at the periphery of the proton. A new frontier in understanding this structure would be possible at CERN within the COMPASS experimental setup. Major advances have already been done on the design of the project and simulation outputs.

\section{References}

[1] F. D. Aaron et al. [H1 Collaboration], Phys. Lett. B 659 (2008) 796; arXiv:0907.5289 [hep-ex].

[2] S. Chekanov et al. [ZEUS Collaboration], JHEP 0905 (2009) 108.

[3] K. Kumericki, D. Mueller and K. Passek-Kumericki, Nucl. Phys. B 794 (2008) 244.

[4] N. d'Hose et al., Nucl. Phys. A 711 (2002) 160. 